\title{
Performance Analysis of DWMT Transceiver for ADSL Channel with Overlap FDE in Presence of AWGN, Crosstalk and Impulse Noise
}

\author{
Arsla Khan, Sobia Baig, and Soo Y. Shin
}

\begin{abstract}
Discrete wavelet Multi-tone (DWMT) was proposed to overcome the problems of Discrete Multi-tone (DMT) for wireline channels. DWMT based system poses more resistance against inter-symbol interference (ISI) because of tight filters and more spectral efficiency due to absence of cyclic prefix as compared to DMT based system. However, no equalization technique has been standardized yet for DWMT transceiver. In this paper, we are proposing Overlap frequency domain equalization (FDE) for DWMT transceiver for ADSL channel in presence of additive white Gaussian noise (AWGN), crosstalk and impulse noise (IN). From simulations results, we can analyze that Bit error rate $(B E R)$ performance of Time domain equalization (TDE) is comparable with Overlap FDE but the main advantage lies in lower computational complexity.
\end{abstract}

Index Terms-ADSL, AWGN, DMT, DWMT, FEXT, impulse noise, NEXT, overlap FDE.

\section{INTRODUCTION}

Orthogonal division multiplexing (OFDM) has come forward as a competent multi-carrier modulation (MCM) technique. It mitigates the effect of frequency selective channels by dividing the entire wide band into small subbands [1]. DMT, which is considered as a variant of OFDM was proposed by Chew and Cioffi for wired channels like Asymmetrical digital subscriber loop (ADSL) signaling [2]. Just like OFDM, DMT uses Inverse Fast Fourier Transform (IFFT) and Fast Fourier Transform (FFT) at transmitting and receiving sides respectively. Although DMT exhibits significant advantages, which have proved its potential in digital communication, however it still has some drawbacks such as redundant cyclic prefix (CP), higher peak to average power ratio (PAPR), and spectral leakage which results in higher side lobes.

To overcome these draw backs, an alternative approach based on discrete wavelet transform (DWT) was proposed [3]. This new scheme which was proposed by Sandberg and Tzannes in 1995 is called DWMT for wired channels [4]. Wavelet transform (WT) based DMT systems are considered spectrally efficient because it does not use CP.

Manuscript received June 5, 2015; revised November 12, 2015. This work was supported part by the Wireless and Emerging Network System Laboratory (WENS Lab) in Kumoh National Institute of Technology, Republic of Korea.

Arsla Khan and Soo Young Shin are with the Department of IT Convergence Engineering, School of Electronic Engineering, Kumoh National Institute of Technology, Gumi, South Korea (e-mail: arslakhan@ciitlahore.edu.pk, wdragon@kumoh.ac.kr).

Sobia Baig is with the Department of Electrical Engineering, COMSATS Institute of Information Technology, Lahore, Pakistan (e-mail: drsobia@ciitlahore.edu.pk).
Spectral containment of channels is better in DWMT as compared to DMT [5]. FFT based systems have high energy containment in side lobes as compared to DWT based systems, which makes DWT systems more robust against narrowband interference and multipath fading affects [6]. WT based MCM technique has been standardized for power line communication (PLC) in IEEE P1901 [7].

Wavelet based filters can reduce ISI but they are not able to eliminate it completely. In order to reduce ISI and undo channel affects, several signal processing techniques may be used, which are collectively known as equalization techniques. Despite all advantages, DWMT based systems require computationally efficient and less complex channel equalization techniques for dispersive channels like ADSL [8]. But problem arises because of the overlapping nature of DWMT symbols in time domain. Few works are available in literature, which proposes various TDE and FDE techniques for wavelet based multi-carrier systems. These techniques are discussed in [9]-[12]. However, the equalization of DWMT modulation technique with less complexity and efficiency is still an open research area. Thus, in this paper, a new technique Overlap FDE proposed by Tomeba, Takeda and Adachi in [13] is applied on DWMT based ADSL channel. To the best of our knowledge, Overlap FDE has not been used for DWMT systems for ADSL downlink channel in presence of AWGN, crosstalk and IN.

In this paper, we have simulated the DWMT transceiver for ADSL channel with TDE and Overlap FDE. Performance of the system is analyzed in presence of AWGN, crosstalk and IN in terms of BER along with comparison in computational complexity. From simulation results, we can deduce that the $B E R$ performance of both equalization techniques is comparable, but the computational complexity of Overlap FDE is much less than the TDE techniques.

This paper is organized into different sections. Section II is about the basic DWMT transceiver structure. Section III describes the ADSL channel along with different channel impairments like cross talk and IN. Section IV presents TDE and Overlap FDE for DWMT transceiver over ADSL channel in detail. Simulation results are shown in Section V. Section VI gives computational complexity comparison of both TDE and overlap FDE techniques whereas Section VII draws the conclusions.

\section{BASIC DWMT TRANSCEIVER STRUCTURE}

The functional block diagram of DWMT transceiver is shown in Fig. 1. Transmitter accepts the data in the form of 
high data rate bit stream which is serial in nature. This serial bit stream is then divided into multiple parallel bit streams of low data rate after passing through serial/parallel converter. During serial to parallel conversion, instead of fixed loading, adaptive bit loading is implemented. These parallel streams are then sent to constellation encoder. Here bit streams are mapped onto QAM constellation according to the number of bits loaded and converted into sequences. These sequences are modulated through WT. The Inverse Discrete Wavelet transform (IDWT) is implemented at the transmitter. With the help of IDWT, not only frequency but also time information can be estimated [14]. Parallel streams are then combined together into serial stream and then sent to receiver through channel.

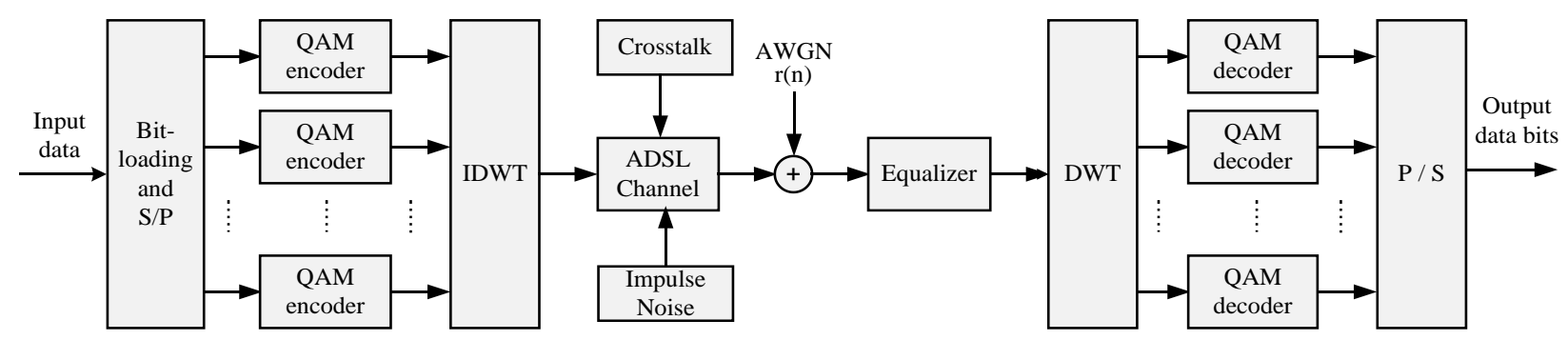

Fig. 1. Block diagram of DWMT transceiver.

On the receiver side, the serial stream is passed through an equalizer to undo the channel effects. After equalizer, the stream is passed through the DWT block. After DWT block, serial stream is again converted back into parallel streams. These parallel streams are then sent to QAM decoder for constellation demapping. After demapping, the sequence is converted back into bits and then these bits of parallel stream are combined together to get the final output bit stream.

\section{Water Filling Adaptive Bit Loading Algorithm}

In communication channels, to perform better in fading environment all sub-channels are loaded with different number of bits which is called adaptive bit loading. One of the its obvious advantage is less probability of error.

In our proposed system, water filling bit loading algorithm is used for bit loading. Bits will be loaded according to the $S N R$ of each sub-channel. First of all it is decided as to which tones are to be turned off due to low $S N R$ values. Bits are then assigned to sub-channels according to the value of $S N R$. These allocated bits are given by the formula [15].

$$
b_{n}=\frac{1}{2} \log _{2}\left[1+\frac{S N R}{\Gamma \cdot \gamma_{m}}\right]
$$

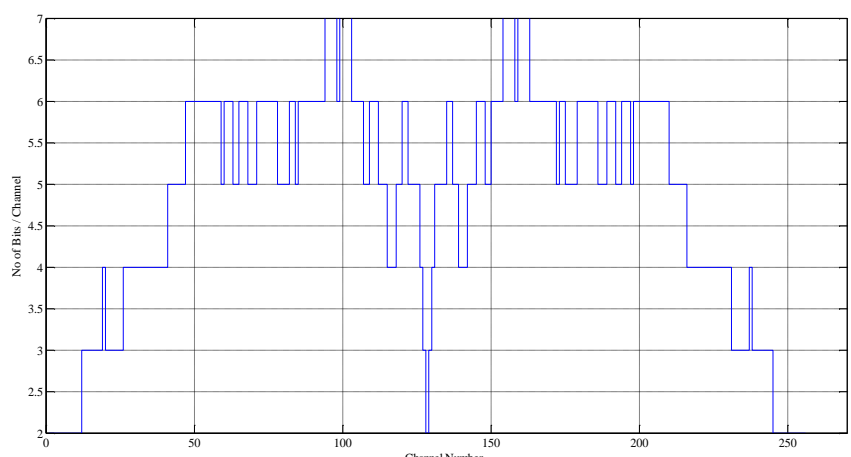

Fig. 2. Variable bit loading on ADSL channel.

where $b_{n}$ is the number of bits on $n^{\text {th }}$ sub-channel, SNR is equal to $\epsilon_{n} \cdot g_{n}$ It is the $S N R$ of each sub-channel. $\epsilon_{n}$ is the sub- channel energy. $g_{n}$ is the gain of sub-channel. $\Gamma$ is $S N R$ gap which presents the difference between channel capacity and target bit rate. $\gamma_{m}$ is performance margin. $g_{n}$ can be calculated by the formula written in (2),

$$
g_{n}=\frac{\left|H_{n}\right|^{2}}{\sigma^{2}}
$$

where $H_{n}$ is the channel and $\sigma^{2}$ is the noise power. A major drawback of water-filling algorithm is rounding off of values which results in performance degradation. This drawback is called granularity [16]. Fig. 2. shows the variable bit loading phenomena for ADSL channel whose impulse response is shown in Fig. 3.

\section{ADSL CHANNEL MODEL WITH CHANNEL IMPAIRMENTS}

ADSL is an asymmetric channel where downstream and upstream data rates are different. Stimulated ADSL channel is a finite Impulse Response (FIR) filter, having length equal to 101 coefficients as shown in Fig. 3. ADSL channels are plagued by many disturbances such as crosstalk, radio frequency interference and IN [17]. Due to these impairments, the rate at which data is to be transmitted gets limited.

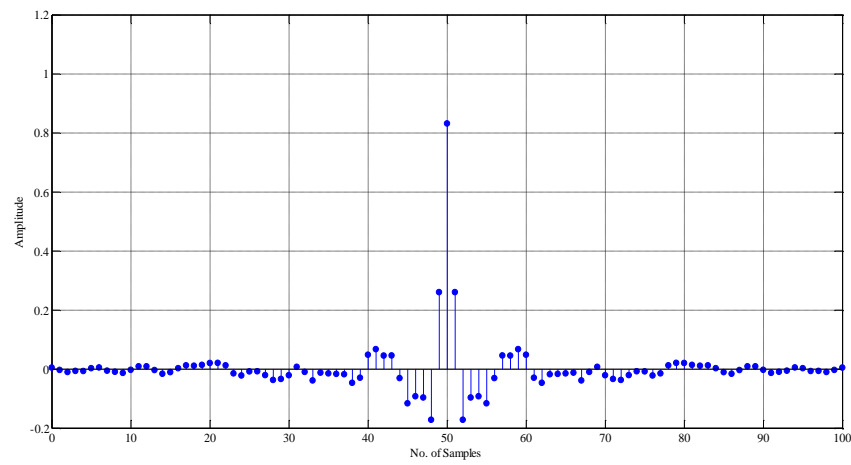

Fig. 3. Impulse response of telephone channel [7]

\section{A. Crosstalk-Capacity Limiting Noise}

Crosstalk is considered as a capacity limiting noise for DSL channels [18]. It generates because of the coupling between different unshielded twisted pairs (UTP). Based on interference location, it can be divided into two types i.e. Far end crosstalk (FEXT) and near end cross talk (NEXT). NEXT coupling is the ability of transmitting signals to 
interfere with signals of other wires within same cables [19]. FEXT appears on another pair at the opposite end or far end of cable as compared to the source of interference. For our simulations, modeling of FEXT and NEXT is performed according to the parameters documented in G.992.1/G.922.2 [20]. These parameters are listed in the Table I.

TABLE I: MODELING PARAMETERS FOR NEXT AND FEXT

\begin{tabular}{ccc}
\hline Parameters & Notations & Values \\
\hline No. of Disturbers & $\mathrm{n}$ & 24 \\
Sampling Frequency & $\mathrm{f}_{\mathrm{s}}$ & $2.208 \mathrm{MHz}$ \\
Low pass cut-off frequency & $\mathrm{f}_{\mathrm{LP3} \mathrm{dB}}$ & $f_{S} / 2$ \\
High pass cut-off frequency & $\mathrm{f}_{\mathrm{HP} 3 \mathrm{~dB}}$ & $138 \mathrm{KHz}$ \\
Coupling constant & $\mathrm{K}_{\mathrm{ADSL}}$ & $0.1104 \mathrm{watts}$ \\
NEXT frequency & $\mathrm{f}_{\mathrm{NXT}}$ & $160 \mathrm{KHz}$ \\
NEXT power sum loss & $\mathrm{NPSL}$ & $47 \mathrm{~dB}$ \\
FEXT frequency & $\mathrm{f}_{\mathrm{FXT}}$ & $160 \mathrm{Khz}$ \\
Distance & $\mathrm{d}_{\mathrm{FXT}}$ & $1.0 \mathrm{~km}$ \\
FEXT power sum loss & FPSL & $45 \mathrm{~dB}$ \\
\hline
\end{tabular}

Power spectral densities (PSD) for disturber noise (downstream), disturber noise (upstream), ADSL downstream NEXT and ADSL downstream FEXT are given below respectively [20],

$$
\begin{gathered}
P S D_{d_{-} D S T}=K_{D S T} \frac{2\left(\sin \left(\frac{\pi f}{f o}\right)^{2}\right)}{\left(f o\left(\frac{\pi f}{f_{o}}\right)^{2}\right)\left(1+\left(\frac{f}{f_{L P 3 d B}}\right)^{12}\right)\left(1+\left(\frac{f_{H P 3 d B}}{f}\right)^{16}\right)}(3) \\
0 \leq f<\infty \\
P S D_{u_{-} D S T}=K_{D S T} \frac{2\left(\sin \left(\frac{\pi f}{f o}\right)^{2}\right)}{\left(f o\left(\frac{\pi f}{f_{o}}\right)^{2}\right)\left(1+\left(\frac{f}{f_{L P 3 d B}}\right)^{16}\right)\left(1+\left(\frac{f_{H P 3 d B}}{f}\right)^{8}\right)} \\
0 \leq f<\infty \\
P S D_{N X T}=P S D_{d_{-} D S T}\left(10^{\left.-\frac{N P S L_{n}}{10} \times f_{N X T}^{-3 / 2}\right) f^{3 / 2}}\right. \\
0 \leq f<\infty \\
P S D_{F X T}=P S D_{d-D S T}\left|H_{C H}\right|^{2}\left(10^{-\frac{F P S L_{n}}{10}} d_{F X T}^{-1} f_{F X T}^{-2}\right) d f^{2}(6) \\
0 \leq f<\infty
\end{gathered}
$$

where $H_{C H}$ is the channel transfer function. $N P S L_{n}$ and $F P S L_{n}$ are power sum losses for NEXT and FEXT for $n$ disturbers.

\section{B. Impulse Noise-Performance Limiting Noise}

IN is a non-stationary interference which consists of energy spikes. These spikes are random in nature with random amplitudes [21]. Different surveys have been performed by telephone companies to study the behavior of IN [22]-[24]. However, it is not possible to present a complete model for IN [17]. Among different models, IN models presented in [25] and [26] are most popular. DMT systems performance in presence of IN is discussed in [27]. Middleton proposed three models for IN [28]. Middleton noise model A is used for the generation of IN in our simulation system, as it was used in [27].

$$
f_{x}(x)=e^{-A} \sum_{k=0}^{\infty} \frac{A^{k}}{k ! \sqrt{2 \pi \sigma_{k}^{2}}} e^{-\frac{x^{2}}{2 \sigma_{k}^{2}}}
$$

where $\sigma_{k}^{2}=\frac{\frac{k}{A}+\Gamma}{1+\Gamma}$ is the noise variance. $A$ is the impulse noise index, where $A$ is defined as [28],

$$
A=v_{t} T_{s}
$$

$v_{t}$ is the mean impulse rate and $T_{s}$ the is mean impulse duration or its length. Class A noise samples can therefore be described as [10],

$$
n=x_{g}+\sqrt{K_{k}} y
$$

where $x_{g}$ represents white Gaussian background noise with mean zero and variance $\sigma_{g}^{2} . K_{k}$ is a Poisson random variable with mean $A . y$ is another Gaussian sequence having zero mean and variance equal to $\frac{\sigma_{l}^{2}}{A}$. Here $\sigma_{l}^{2}$ is the variance of impulse noise. Cross talk and impulse noise degrades ADSL system performance so to improve it, an appropriate equalization technique is required.

\section{EQUALIZATION TECHNIQUES FOR DWMT TRANSCEIVER OVER ADSL CHANNEL}

Inter block interference (IBI) and Inter Carrier Interference (ICI) is a big problem in delivering high data rates. Receivers have equalizer filters that are designed to compensate IBI and ICI. Channel equalization has been studied to improve the performance of filter banks based communication system by combating ISI [29].

In DWMT based systems, design of an optimal equalization technique is challenging because of the overlapping nature of DWMT symbols in time domain. Thus, it is necessary to avoid IBI and ICI for distortion-less and error free communication [30]-[32]. Though different equalization techniques have been examined in the past for DWMT based systems but a vigorous equalization technique with less complexity is still need to be standardized.

\section{A. Time Domain and Frequency Domain Equalization}

In DWMT transceiver, to combat IBI, TDE equalization has to be performed separately for each sub-channel. However, one of the main drawbacks of TDE approach is high complexity. As the length of channel increases, complexity of the equalizer also increases.

FDE is used in those cases where channel delay spread is very large. In case of large delay spread, TDE equalizer structure becomes complex, thus FDE is preferred because of its less complexity as compared to TDE [7].

\section{B. Overlap Frequency Domain Equalization}

To overcome the complexity of TDE techniques and inefficiency due to guard interval (GI) insertion of FDE techniques, a new technique overlap FDE was proposed.

Overlap FDE does not need any GI and improves the bandwidth efficiency of the system. For the first time, Overlap FDE was applied by Tomeba for MCM systems [13] and later used by Sohaib for wavelet OFDM [13]. In [7], authors showed its supremacy for ADSL systems for DWMT transceiver in presence of AWGN and FEXT, NEXT. In this paper, we are evaluating Overlap FDE performance for ADSL systems for DWMT transceiver in 
presence of AWGN, FEXT, NEXT and impulse noise.

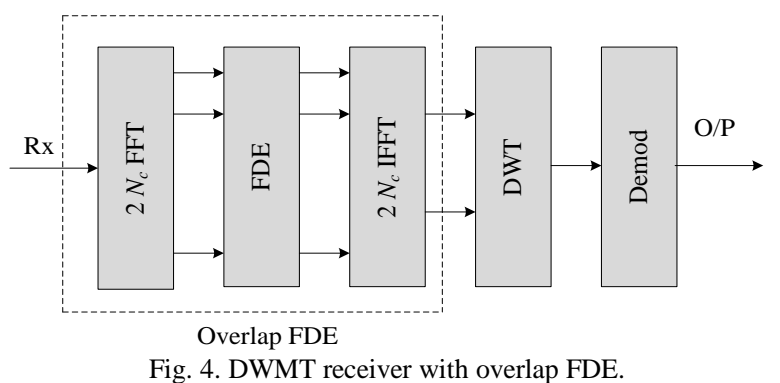

DWMT transceiver with Overlap FDE is shown in Fig. 4. At the receiver side, the received signal $R_{x}$ is first passed through the block of $2 N_{c}$ point FFT, where $N_{c}$ is the number of sub-carriers used. Received signal $r_{m}(t)$ is in the time domain. After passing through FFT block, time domain signal $r_{m}(t)$ is converted into frequency domain signal $R_{m}(q)$. Received signal in frequency domain is given as [13],

$$
R_{m}(q)=\frac{1}{2 N_{c}} \sum_{t=0}^{2 N_{c}-1} r_{m}(t) \exp \left(-j 2 \pi q \frac{t}{2 N_{c}}\right)
$$

Here the value of $q$ varies from 0 to $2 N_{c}-1 . R_{m}(q)$ can also be written in form of

$$
R_{m}(q)=H(q) Y_{m}(q)+N_{m}(q)+\Pi_{m}(q)
$$

Here, $Y_{m}(q)$ is the transmitted signal passing through the channel $H(q)$. During its transmission through channel, it gets affected due to IBI and AWGN. $N_{m}(q)$ is the AWGN added in the signal while $\Pi_{m}(q)$ is the IBI factor. In our case $N_{c}=256$. Thus, due to $2 N_{c}$ point FFT, the received signal is decomposed into 512 frequency components for $N_{c}$ $=256$ sub-carriers. Equalization is performed according to the taps optimized by either zero forcing (ZF) and minimum mean square error (MMSE). FDE weights $c(q)$ are calculated by method given in [7].

$$
c(q)=\left\{\begin{array}{lr}
\frac{H^{*}(q)}{|H(q)|^{2}} & Z F \\
\frac{H^{*}(q)}{|H(q)|^{2}+\left(\frac{E_{\text {avg }}}{N_{O}}\right)^{-1}} & M M S E
\end{array}\right.
$$

where $H(q)$ is the channel impulse response. $E_{a v g}$ is the average energy per symbol while $N_{o}$ is the AWGN. Equalized signal can be represented like,

$$
R^{\prime}(q)=R(q) c(q)
$$

Equalized signal $R^{\prime}(q)$ is then passed through $2 N_{c}$ point IFFT which will convert the frequency domain signal into time domain signal.

$$
\widehat{r}_{m}(t)=\sum_{q=0}^{2 N_{c}-1} R^{\prime}(q) \exp \left(j 2 \pi q \frac{t}{2 N_{c}}\right)
$$

The original length of our data was 256 but due to $2 N_{c}$ point FFT and IFFT, it becomes double. We know that since IBI is present at the edges of all blocks so if we discard the samples present at corners of the block then IBI can be removed. Thus, IBI present at the corners are suppressed by simply removing the 256 samples present at the edges and by picking the central 256 samples. This data is then sent to the block of DWT. Demodulation is performed at the received data and then original signal is retrieved.

\section{Simulation Results}

DWMT transceiver is designed and simulated in MATLAB. Channel impairments like AWGN, FEXT, NEXT and impulse noise are introduced in DWMT system. TDE and overlap FDE are used to overcome the affects of channel spreading. For this purpose equalizer performance is analyzed with the help of two tap optimization methods i.e. ZF and MMSE. It is assumed that channel is known and there is no need of channel estimation. Perfect synchronization is considered between transmitter and receiver. Performance of both techniques is compared on the basis of $B E R$ and computational complexity. Detail of simulation parameters is given in Table II.

\begin{tabular}{lc}
\multicolumn{2}{c}{ TABLE II: MODELING SIMULATION PARAMETERS } \\
\hline \multicolumn{1}{c}{ Parameters } & Values \\
\hline Channel Bandwidth & $1.104 \mathrm{MHz}$ \\
Sampling Frequency & $2.208 \mathrm{MHz}$ \\
No. of Sub-channels & 256 \\
Symbol Length & 256 \\
Symbol Period & $0.115942 \mathrm{msec}$ \\
Symbol Rate & $8625 \mathrm{symbols} / \mathrm{sec}$ \\
Channel Frequency Spacing & $4.3125 \mathrm{KHz}$ \\
No. of bits per sub-channel & $1 \mathrm{to} 7$ \\
Modulation Scheme & $M-Q A M$ \\
Wavelet Level & 2 \\
Wavelet Family & Haar \\
Equalization Technique & TDE-ZF \\
& TDE-MMSE \\
& Overlap FDE - ZF \\
\hline \hline
\end{tabular}

\section{A. Comparison of TDE-ZF and TDE-MMSE with AWGN, FEXT NEXT and IN}

Performance analysis regarding $B E R$ between TDE - ZF and TDE - MMSE for DWMT transceiver is performed and shown in Fig. 5. Simulations are performed in presence of AWGN, FEXT, NEXT and impulse noise simultaneously. Impulse noise index $A$ is kept constant at 0.0001 . For a $B E R$ of 5.68E-3, TDE - MMSE algorithm has $\frac{E_{b}}{N_{o}}=22 \mathrm{dBs}$ while $\mathrm{ZF}$ has $\frac{E_{b}}{N_{o}}=24 \mathrm{dBs}$. It shows TDE - MMSE equalization algorithm outperforms with a gain of $2 \mathrm{dBs}$ as compared to $\mathrm{TDE}-\mathrm{ZF}$.

\section{B. Comparison of Overlap FDE-ZF and Overlap FDE- MMSE with AWGN, FEXT NEXT and IN}

In this sub-section, simulations are performed on DWMT transceiver for ADSL channel with overlap FDE. Fig. 6 shows that if we fix $B E R$ equal to $1 E-3, \mathrm{ZF}$ technique gives $\frac{E_{b}}{N_{o}}$ equal to $32 \mathrm{dBs}$ while MMSE outperform ZF criteria and we are getting a gain of $6 \mathrm{dBs}$.

If we compare these results with TDE results, we can easily find that for TDE - MMSE $\frac{E_{b}}{N_{o}}$ has value equal to 28 $\mathrm{dBs}$ for $B E R$ of $1 E-3$, whereas in Overlap FDE, the same value of $B E R$ is achieved at $26 \mathrm{dBs}$. It means with MMSE- 
Overlap FDE, we are getting $2 \mathrm{dBs}$ gain as compared to MMSE-TDE.

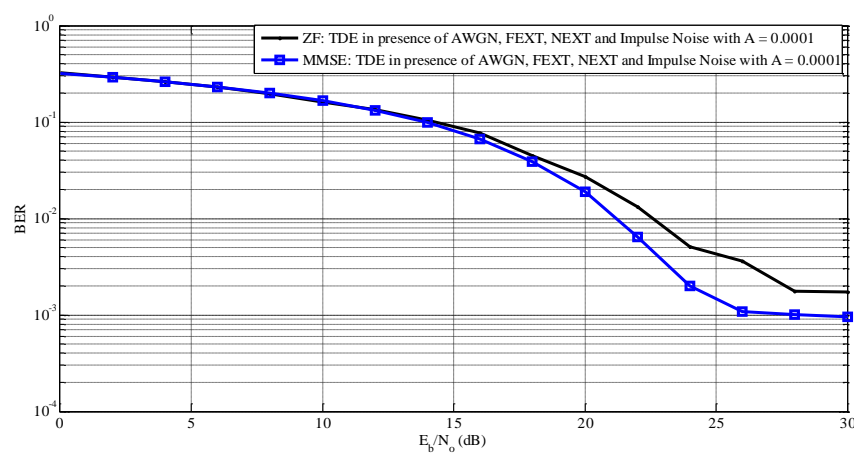

Fig. 5. BER comparison of TDE-ZF and TDE-MMSE for DWMT transceiver for ADSL channel in presence of AWGN, FEXT NEXT and IN with impulse noise index, $A=0.0001$.

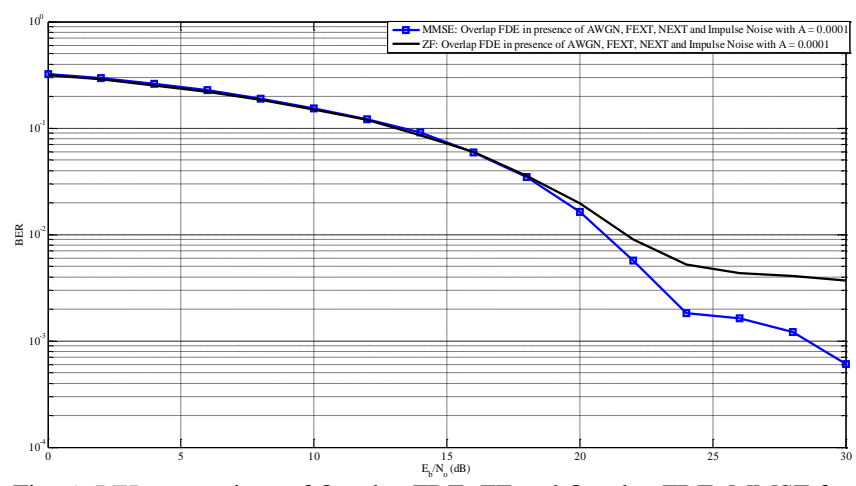

Fig. 6. BER comparison of Overlap FDE-ZF and Overlap FDE-MMSE for DWMT transceiver for ADSL channel in presence of AWGN, FEXT NEXT and IN with $A=0.0001$.

\section{COMPUTATIONAL COMPLEXITY COMPARISON}

In DWMT based system, computation complexity is $O(N)$ where $N$ is the length of the signal. For TDE, the complexity is given by, $O\left(N^{3}\right)$ [7]. Here $N$ is dimension of the signal. For Overlap FDE, the complexity of $2 N_{s}$ points is given by, $\left[2\left(2\left(N_{S} \log _{2} N_{S}\right)\right)+2 N_{S}\right] . N_{S}$ represents the size of IFFT/FFT [7]. Complexity comparison for TDE and Overlap FDE for DWMT transceiver is shown in Table III.

TABLE III: COMPLEXITY COMPARISON OF TDE AND OVERLAP FDE FOR DWMT TRANSCEIVER

\begin{tabular}{lcc}
\hline Equalization Technique & TDE & Overlap FDE \\
\hline $\begin{array}{l}\text { Complexity of } \\
\text { equalization technique }\end{array}$ & $O\left(N^{3}\right)$ & {$\left[2\left(2 N_{s} \log _{2} 2 N_{s}\right)\right.$} \\
System Complexity & $O(N)$ & $\left.+2 N_{s}\right]$ \\
Total Complexity & $O\left(N^{3}\right)+$ & $O(N)$ \\
& $O(N)$ & {$\left[2\left(2 N_{s} \log _{2} 2 N_{s}\right)+\right.$} \\
& $=O(256)+$ & $=O(256)+[(2 \times$ \\
If $N$ and $N_{s}=256$ & $O\left(256^{3}\right)$ & $\left.256 \log _{2} 2 \times 256\right)+$ \\
& $=16777472$ & $2 \times 256]=5376$ \\
\hline
\end{tabular}

\section{CONCLUSIONS}

DWMT based systems have proved incredible advantages over its counterpart DMT based systems in terms of lower side lobes, resistance against IBI and ICI due to its spectral containment. However, its equalization is not easy task because of complexity which comes due to overlapping of DWMT symbols in time domain. In this paper, overlap FDE is proposed for the first time for DWMT transceiver in presence of AWGN, FEXT, NEXT and impulse noise. Results obtained from simulating the system on MATLAB shows that BER performance of both system is comparable. However, supremacy of overlap FDE lies in its lower computational complexity. Therefore, overlap FDE is a good option to equalize DWMT transceiver even in the presence of different types of noises. For future work, authors are interested to apply overlap FDE for power line communication channel for smart grid communications.

\section{ACKNOWLEDGMENT}

This research was supported by the MSIP (Ministry of Science, ICT and Future Planning), Korea, under the CITRC (Convergence Information Technology Research Center) support program (IITP-2015-H8601-15-1011) supervised by the IITP (Institute for Information \& communications Technology Promotion)

\section{REFERENCES}

[1] H. G. Myung, "Introduction to single carrier FDMA," in Proc. the 15th European Signal Processing Conference (EUSIPCO), Sep. 3-7, 2007, pp. 2144-2148.

[2] P. S. Chow, J. C. Tu, and J. M. Cio, "Performance evaluation of a multichannel transceiver system for ADSL and VHDSL services," IEEE Journal on Selected Areas in Communication, vol. 9, no. 6, pp. 909-919, Aug. 1991.

[3] K. Abdullah and Z. H. Hussain, "Impulse noise effects on DWT- and WPT-OFDM versus FFT-OFDM," in Proc. International Conference on Communication, Computer and Power, Feb. 2009, pp. 373-377.

[4] G. Jovanovic-Dolecek, Multirate Systems: Design and Application, United States of America: Idea Group Publishing (IGP), Feb. 1, 2002.

[5] R. Dilmirghani and M. Ghavami, "Wavelet Vs. Fourier based UWB systems," in Proc. the IEEE 18th IEEE International Symposium on Personal, Indoor and Mobile Radio Communications, 2007, pp. 1-5.

[6] S. Baig, F. Rehman, and M. J. Mughal, "Performance comparison of DFT, discrete wavelet packet and wavelet transforms, in an OFDM transceiver for multipath fading channel," in Proc. the IEEE 9th International Multitopic Conference, 2005, pp. 1-6.

[7] A. Khan, S. Baig, and T. Nawaz, "DWMT transceiver equalization using overlap FDE for downlink ADSL," Turkish Journal of Electrical Engineering \& Computer Sciences, vol. 23, pp. 681-697, 2015.

[8] F. Farrukh, "Discrete wavelet packet multi-tone based transceiver for asymmetric digital subscriber line," M.S. thesis, GIKI Institute of Engineering Sciences and Technology Topi, Pakistan, May 2007.

[9] S. A. Elghafar, T. M. Halim, S. M. Diab, and B. M. Sallam, "New equalization approach for maximizing data rate in discrete multi-tone systems," in Proc. the $28^{\text {th }}$ National Radio Science Conference, April 26-28, 2011, pp. 1-11.

[10] S. Baig and M. J. Mughal, "A frequency domain equalizer in discrete wavelet packet Multi-tone transceiver for in-home PLC lans," in Proc. the IEEE International Symposium on Power Line Communications and Its Applications, 2007, pp. 181-186.

[11] L. Zhou, J. Li, and P. He, "Novel WPDM system based on channel equalization for multipath fading channels," in Proc. the $20^{\text {th }}$ International Conference on Advanced Information Networking and Applications, April 18-20, 2006, pp. 909-914.

[12] D. Cariolaro and L. Favalli, "Recovery of ISI channels using multiresolution wavelet equalization," in Proc. the IEEE International Conference on Communications, 2002, vol. 1, pp. 74-78.

[13] H. Tomeba, K. Takeda, and F. Adachi, "BER performance analysis of MC-CDMA with Overlap-FDE," IEICE Transactions on Communications, vol. E91-B, no. 3, pp. 795-804, 2008.

[14] N. Neurohr and M. Schilpp, "Comparison of trans-multiplexers for multicarrier modulation," in Proc. the Fourth International Conference on Signal Processing, 1998, vol. 1, pp. 35-38.

[15] W. Yu and J. M. Cioffi, "On constant power water-filling," in Proc. the IEEE International Conference on Communications, 2001, vol. 6, pp. 1665-1669.

[16] S. Panigrahi and T. Le-Ngoc, "Fine-granularity loading schemes using adaptive reed-Solomon coding for xDSL-DMT systems," European Association for Signal Processing Journal on Applied Signal Processing, 2006. 
[17] F. H. Gregorio, J. E. Cousseau, and J. L. Figueroa, "Reducing impulse noise in DSL systems - Robustness and delay," in Proc. the 6th Nordic Signal Processing Symposium, June 2004, pp. 224-227.

[18] J. W. Cook, R. H. Kirkby, M. G. Booth, K. T. Foster, D. E. A. Clarke, and G. Young, "The noise and crosstalk environment for ADSL and VDSL systems," IEEE Communications Magazine, vol. 37, no. 5, pp. 73-78, May 1999.

[19] T. Starr, J. Cioffi, and P. Silverman, Understanding Digital Subscriber Line Technology, Upper Saddle River, NJ: Prentice Hall, 1999.

[20] K. V. Padmaja, B. V. Uma, and S. Ravishankar, "Simulation and implementation of a practical ADSL environment on DSP Kit," World Academy of Science, Engineering and Technology, pp. 22-27, 2007.

[21] R. Dragomir, S. Puscoci, and D. Dragomir, "A synthetic impulse noise environment for DSL access networks," in Proc. the 2nd International Conference on Circuits, Systems, Control, Signals, Sep. 2011, pp. 116-119.

[22] D. Levey and S. McLaughlin, "Statistics of impulsive noise: Interarrival times," ETSI WG TM6, TD19, 993T19A0, 1999.

[23] W. Henkel, T. Kessler, and H. Y. Chung, "Coded 64-CAP ADSL in an impulsive noise environment - modeling of impulsive noise and first simulation results," IEEE Journal on Selected Areas of Communications, vol. 13, no. 9, Dec. 1995.

[24] W. Henkel and T. Kessler, "Statistical description and modeling of impulsive noise on the German telephone network," Electronics Letters, vol. 30, no. 6, pp. 935-936, June 1994.

[25] I. Mann, S. McLaughlin, W. Henkel, R. Kirby, and T. Kessler, "Impulse generation with appropriate amplitude, length, inter-arrival, and spectral characteristics," IEEE Journal on Selected Areas in Communications, vol. 20, no. 5, pp. 901-912, June 2002.

[26] S. N. Batalama, M. J. Medley, and I. N. Psaromiligkos, "Adaptive robust spread-spectrum receivers," IEEE Transactions on Communications, vol. 47, no. 6, pp. 905-917, June 1999.

[27] G. Pay and M. Safak, "Performance of DMT systems under impulsive noise," in Proc. the International Symposium on Power Line Communications, 2001, pp. 104-109.

[28] S. A. Bhatti, Q. Shan, I. A. Glover, R. Atkinson, I. E. Portugues, P. J. Moore and R. Ruthford, "Impulsive noise modeling and prediction of its impact on the performance of WLAN receiver," in Proc. the 17th European Signal Processing Conference, Aug. 2009, pp. 1680-1684.

[29] E. F. Badran, "Optimum channel equalization for filterbank transceivers in presence of white noise," PhD thesis, Louisiana State University, May 2002.
[30] M. Hawryluck, "Evaluation of modulation techniques for broadband access over twisted pair," M.Sc. thesis, University of Ottawa, June 1997.

[31] S. Kiyani, S. Baig, and M. J. Mughal, "Overlap frequency domain equalization for wavelet OFDM," in Proc. the International Technical Conference on Circuits/Systems, Computers and Communications, July 2009, pp. 1346-1349.

[32] J. P. Wilkinson, "Nonlinear resonant circuit devices," U.S. Patent 3 624 12, July 16,1990

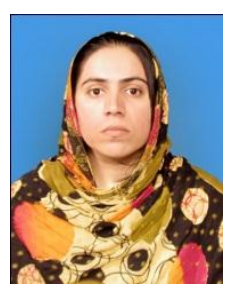

Arsla Khan was born in Lahore, Pakistan. She received the B.Sc. and M.Sc. degrees from COMSATS Institute of Information Technology (CIIT), Lahore, Pakistan in electrical engineering in 2008 and 2012, respectively. She is currently pursuing the Ph.D. degree with the Department of IT Convergence Engineering, School of Electronic Engineering, Kumoh National Institute of Technology (KIT), South Korea. Her research interests include multi-rate communication networks and $5 \mathrm{G}$

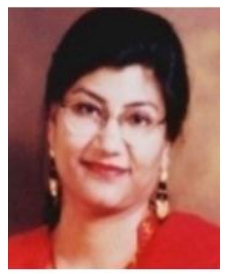

Sobia Baig received her B.Sc. degree in electrical engineering from University of Engineering and Technology, Taxila, Pakistan in 1995. She received her masters and $\mathrm{PhD}$ degrees from the Faculty of Electronic Engineering, GIKI, Topi, Pakistan in 2003 and 2008, respectively. Currently she is working as an associate professor and the head of EE Department in CIIT. Her research interests include MCM techniques.

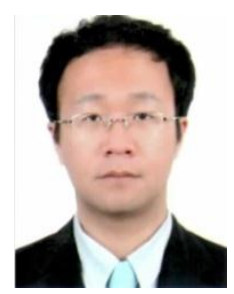

Soo Young Shin was born in 1975 . He received his B.S., M.S., and Ph.D. degrees in electrical engineering and computer science from Seoul National University, Korea in 1999, 2001, and 2006 , respectively. His research interests include cognitive radio networks, MIMO, OFDM, NOMA and next generation mobile wireless broadband networks $(4 \mathrm{G} / 5 \mathrm{G}) . \mathrm{He}$ is an assistant professor in the School of Electronics in KIT since September 2010. 